\title{
ORÍGENES DE LA INDUSTRIA PESQUERA PERUANA
}

\author{
Maria Elizabeth Puertas Porras \\ Héctor Maldonado Félix
}

\section{Industria Pesquera Perú-EE. UU. Siglo XX}

La revolución industrial que se inició en el siglo XVIII produjo un gran crecimiento demográfico que presionó los sectores productores de alimentos. Esta presión llevó a que el sector pesquero emplee la red de enmalle pelágica desde la segunda mitad del siglo XIX, la cual permitió incrementar la extracción de pescado. La demanda urbana impulsará la explotación del atún en el siglo XX.

El atún, familia Scombridae y género Thunnus, se diferencia en 8 especies, destacando por su consumo el Thunus albacares (Yellowfin), T. alalunga (Albacore), T. orientalis (Bluefin) y Katsuwuono pelamis (Skipjack). Estas especies miden de entre 1 a 2 metros de largo y llegan a pesar entre 33 a $175 \mathrm{~kg}$. El atún vive en aguas desde $18^{\circ} \mathrm{C}$ a $10^{\circ} \mathrm{C}$ y es un pez migrante, pelágico y se desplaza en cardúmenes de 3000 miembros. Para capturarlo se emplea como carnada a la anchoveta. ${ }^{1}$

1 Véase <www.fao.org/fishery/topic/16082/en> (consultada el 19 de marzo de 2009). 


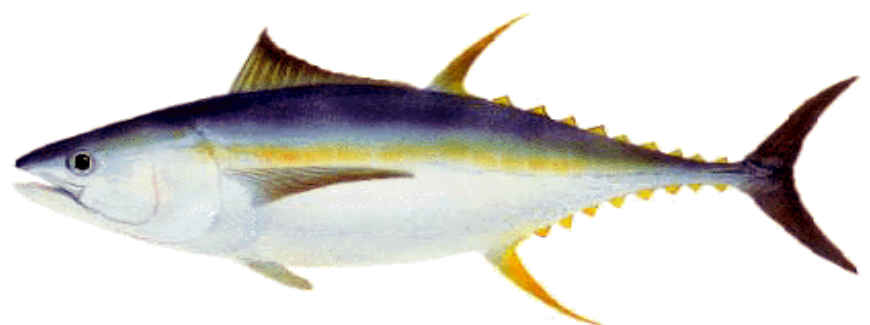

Yellowfin

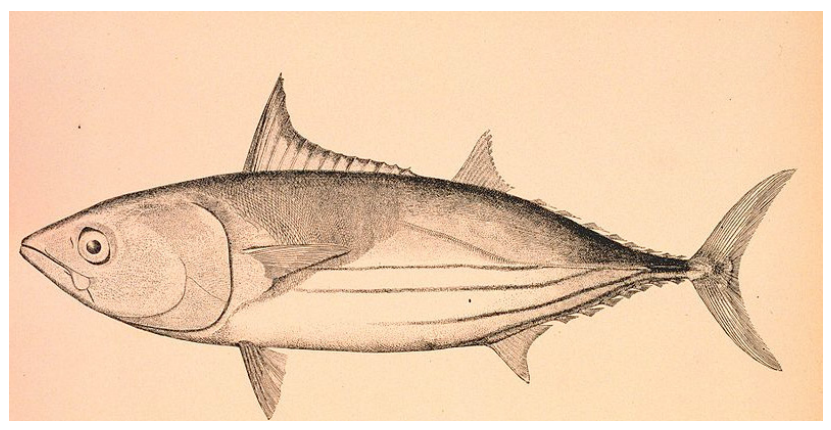

Skipjack

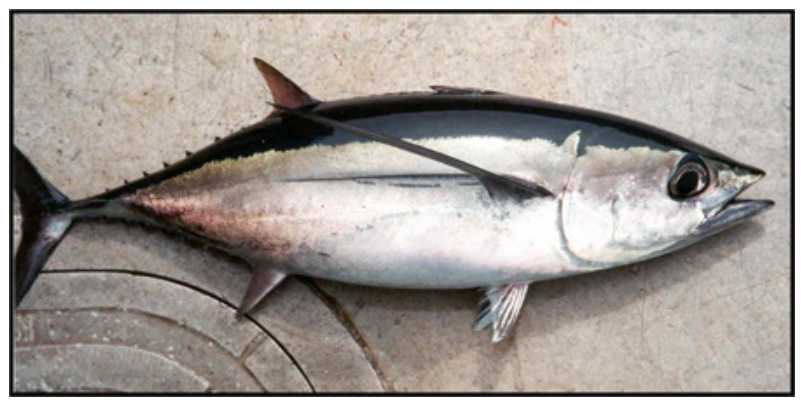

Albacore 
INDUSTRIA PESQUERA DE EE. UU.

La conservación de alimentos se inició en Francia en el siglo XIX. Hacia 1822 ya se comercializaban conservas en Inglaterra, Francia y EE. UU. En el siglo XIX surgen las primeras conserveras en EE. UU. como en Eastport, Maine (1875). A fines del siglo XIX la industria pesquera inicia la explotación de la costa Oeste orientándose primero al salmón en las costas del estado de Washington y, posteriormente, al atún en las costas del estado de California. En principios del siglo XX - 1903 - se inició la industria conservera de atún en aceite.

El impacto de la pesquería sobre el recurso marino llevó a EE. UU. a crear en 1871 la U.S. Commission of Fish and Fisheries y en 1903 la U.S. Bureau of Fisheries, que convirtieron a la naciente oceanografía en una herramienta de la diplomacia de EE.UU en el siglo XX (cf. Finley 2009).

En el siglo XX, el estado de California tenía una envidiable posición económica, una creciente población migrante con gran experiencia laboral, acceso a energía barata como la hidroeléctrica y la petrolera.

Las ciudades de San Francisco, Los Angeles y San Diego se destacarán en este sector industrial. Las principales compañías fueron la California Fish Company (1893), Bumble Bee (1899), Van Camp Sea Food Company (1914) y la French Sardine Company más tarde llamada Star Kist (1917).

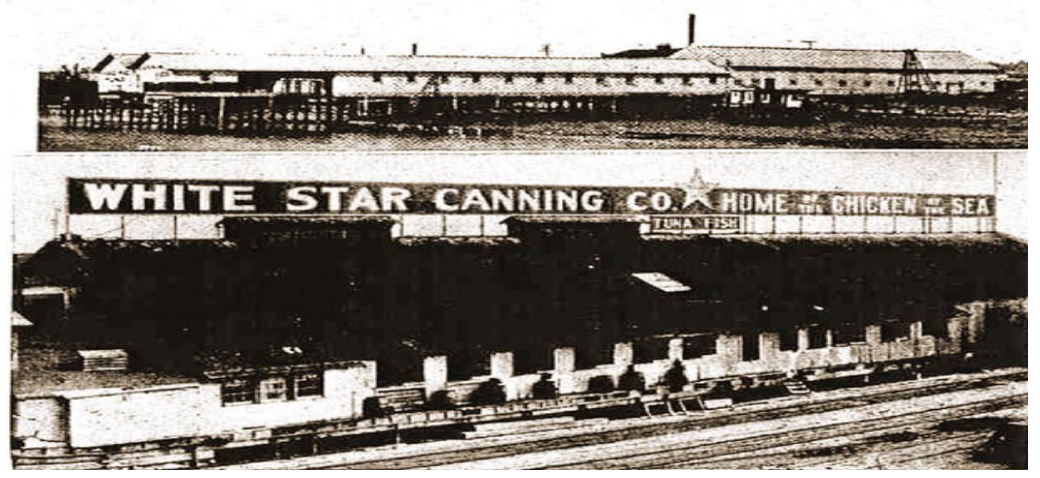

Pacific Tuna Company (1911) y White Star Canning (1912) 
En California se extrajo 16300 Tm de atún y 69400 Tm de sardina en 1919, y $24305 \mathrm{Tm}$ de atún y $143316 \mathrm{Tm}$ de sardina en 1925. El número de barcos pasó de 73 en 1915 a 362 en 1925 que comienzan a emplear gasolina desde 1910 y el número de trabajadores pasó de 2100 en 1918 a 4518 en 1925 (Macevoy 1983: 499).

La industria pesquera que se inició en Europa se extendió a EE. UU. y Japón. En este último país, a diferencia de EE.UU., el mercado se caracterizó por el consumo de conservas mientras en Japón se orientó al consumo de pescado fresco (Finley: 2007: 170).

En la década de 1910, la industria pesquera se diversificó al comenzar a instalarse fábricas de harina de pescado junto a la fábrica de conservas. La producción de harina de pescado, empleada como abono y comida de aves, pasó de 482 Tm en 1916 a 9000 Tm en 1919. En la década de 1920, la empresa dominante era la Wilbur-Ellis, de gran importancia en el Perú, fundada en San Francisco (Macevoy 1983: 504).

A pesar de una serie de leyes que promulgó el Estado de California (1919 y 1921) y de las agencias federales encargadas de los recursos marinos, se continuó con la sobreexplotación de los recursos marinos obligando a reemplazar desde 1919 al Thunus alalunga por el yellowfin y el skipjack (barrilete) y desde la década de 1920 pescar en aguas mexicanas (Macevoy 1983: 507).

La primera crisis que vivió esta industria norteamericana se produjo después de la Primera Guerra Mundial pues de 57 fabricas en 1919 sólo quedaron 34 en 1923, cayendo también el empleo un 55\% y la flota pesquera quedó bajo dominio de las empresas de conservas (Macevoy 1983: 510). La industria era dominada por las fábricas de conservas que daban créditos a los propietarios de los barcos de pesca hacia 1920-1930. En la década de 1920 la industria conservera se concentra en dos especies yellowfin y skipjack que eran capturados desde México hasta el Perú.

En las primeras décadas del siglo XX, Japón aparece como un duro competidor de la industria norteamericana. Japón tenía una cultura orientada hacia la pesca desde hace milenios, tradición que las reformas de 
la era Meiji no cambiaron. En 1899, el estado promovió el desarrollo de una pesca del salmón en alta mar. En las primeras tres décadas del siglo XX, el Japón consolidó su dominio marítimo gracias a su victoria sobre China y especialmente Rusia (1904-1905). La derrota en la Segunda Guerra Mundial afectó seriamente el dominio marítimo japonés.

En 1924, Japón comenzó a exportar el atún albacore congelado al desaparecer éste de las costas de EE.UU., y en 1931 comenzó a exportar conservas de atún. Ante las importaciones japonesas, la caja de conservas del Japón costaba \$3.55 mientras la de EE.UU. valía \$ 5.08. EE. UU. estableció en 1930 una tarifa de $30 \%$ a las conservas que en 1934 fue elevada a 45 \% reduciendo así la importación a la mitad. Durante la Segunda Guerra Mundial, EE. UU., ante la demanda creada, alentó la importación (Petit 1966: 283 y Finley: 2007:142).

La industria pesquera de EE. UU. agotó la sardina en California y, tras una captura de 800000 Tm entre 1936 y 1937, la industria colapsó en 1947, por lo cual se vendieron las fábricas de harina de pescado a Perú. (Macevoy 1990: 199)

Después de la Segunda Guerra Mundial se registró un aumento del mercado mundial y EE. UU. aumentó sus importaciones que pasaron del 7,5\% en 1947 al 34\%. Las principales importaciones lo constituyen el pescado congelado que pasó de 4500 Tm en 1947 a 70000 Tm en 1957, provenientes principalmente de Japón y compradas por las empresas conserveras de California. La importación de conservas creció de 7000 Tm en 1951 a 20 000 Tm en 1957, que debido a la política comercial de EE. UU. pasó de conservas en aceite a conservas en salmuera que pagaban menos derecho. La importación norteamericana estaba controlada por las conserveras French Sardine Co., Van Camp Sea Food Co. y Bumble Bee que controlaban más de la mitad de la producción de EE.UU (Petit 1966: 276).

Ante la escasez del recurso marino las empresas se extendieron hacia Puerto Rico, Samoa Americana (Océano Pacífico) y Perú. Desde 1950, 37 de los 210 barcos que contaba la flota de pesca industrial se trasladaron a Puerto Rico y Perú (Petit 1966: 284). 
Después de la Segunda Guerra Mundial se expandió el mercado de productos marinos en EE.UU., que ante sus recursos marinos debilitados por la sobre explotación progresivamente se abastecerán de países extranjeros pero lograran explotar recursos marinos a miles de millas de sus bases en California aprovechando la prácticamente inexistente flota pesquera latinoamericana, en el Pacífico salvo Japón o Canadá no tenían competidores. Así desde la década de 1950 la experiencia y maquinaria de la industria pesquera de California será transmitida a distantes lugares como Puerto Rico, Perú y la Samoa Americana.

\section{EMPRESAS PESQUERAS PERUANAS}

En la década de 1920 se planteó un sindicato peruano-norteamericano para establecer una fábrica de conservas en Ilo (Thorp y Bertram 1988: 271). En 1934 se estableció la primera fábrica de conservas. A fines de la década de 1930 había 3 a 4 empresas (Thorp y Bertram 1988: 271).

El Estado peruano entre las décadas de 1940 y 1950 presentaba dentro del predominio de una oligarquía agroexportadora una política comercial que pasó de ejercer controles a la exportación a una ortodoxia liberal.

En el primer gobierno de Prado, en 1941, se gravó con un impuesto la exportación del atún, bonito y shipjack congelados (Ley 9506). Esta situación se renovó en 1946 (Ley 10545). Los derechos que gravaban a los productos pesqueros eran los siguientes:

a) Atún congelado: $10 \%$ sobre la diferencia entre el costo en puerto peruano fijado en 50 dólares y el precio en EE.UU. (costa del Océano Pacífico) menos el importe del flete y del seguro.

b) Bonito y Shipjack pagarán el 10\% sobre la diferencia entre el costo en puerto peruano fijado en 40 dólares y el precio en EE.UU. (Costa del Pacífico) menos el importe del flete y del seguro. 
c) Hígado de pescado: 10 dólares por tonelada métrica

En 1943 se creó el Ministerio de Agricultura que tomaría a su cargo las actividades pesqueras. En estos años la Compañía Administradora del Guano buscaba limitar el impacto de la pesca en la vida de las aves guaneras y prohibir la explotación de la anchoveta.

La expansión de la pesca industrial hizo necesario el abastecimiento de hojalata comprada en EE.UU. acciones que la embajada peruana en Washington facilitará. El Perú tenía consulados en New York, Seattle, New Orleáns, San Francisco y Los Ángeles ciudades de destino de las exportaciones pesqueras

Iniciando la década de 1940, el gobierno de Prado, a partir de un informe técnico americano, promulgó un Decreto para destinar recursos a la adquisición de planta de harina de pescado que se compró en EE.UU., estando bajo control de la Compañía Administradora del Guano (cf. Memoria 1950: 4).

La industria pesquera, a consecuencia del mercado norteamericano, se orientó a la exportación de pescado enlatado y aceite de hígado de pescado que fueron demandados debido al esfuerzo bélico de los aliados en la Segunda Guerra Mundial. El pescado que se exportaría sería el bonito (Roemer 1970: 82).

En esta temprana etapa tenemos en el Perú desde 1944 a la empresa norteamericana Wilbur-Ellis de San Francisco que se dedicaba al área de aceite y harina de pescado. (Thorp y Bertram 1988: 272 y Contreras y Cueto 2007: 266). Entre las peruanas destacó la Industrial Pesquera S.A. (1942), fundada en el Callao, que tuvo el financiamiento del Banco Popular (Thorp y Bertram 1988: 272). La producción de pescado enlatado alcanzaba las $6000 \mathrm{Tm}$ entre 1945-1949 (Klaren 2004: 369).

En el Anuario de Comercio Exterior de 1944 que informa sobre 1943 y 1944 se consignan las siguientes empresas dedicadas a la pesca industrial. 
Empresas pesqueras 1943-1944

\begin{tabular}{|l|l|}
\hline Harina de Pescado & Callao \\
\hline Luis Carrillo & Lima \\
\hline Compañía Nacional de Pesca Sociedad Anónima & Callao \\
\hline Castañón Demetrio & Callao \\
\hline Empresa Pesquera Chalaca & Callao \\
\hline Industria Pesquera Sociedad Anónima & Callao \\
\hline Van Camp Sea Food Inc & Lima \\
\hline Wilbur Ellis & \multicolumn{2}{|l|}{} \\
\hline Pescado en conservas & Lima \\
\hline Compañía Pesquera San Andrés & Callao \\
\hline Dittbon Eugenio & Callao \\
\hline Industria Pesquera Sociedad Anónima & Lima \\
\hline Locro Domingo & Lima \\
\hline Milne y Co. & Callao \\
\hline Noriega Santiago & \multicolumn{2}{|l|}{} \\
\hline Pescado fresco salado & Callao \\
\hline Aguilar y Cia Abel & Callao \\
\hline Carrillo Luis & Callao \\
\hline Compañía de Pesca San Pedro & Callao \\
\hline Empresa Pesquera Chalaca & Callao \\
\hline San Lorenzo Sociedad Anónima & Lima \\
\hline Wilbur - Ellis & \multicolumn{2}{|l}{} \\
\hline Tripas de Pescado & Callao \\
\hline Luis Carrillo & Callao \\
\hline Empresa Pesquera Chalaca & \\
\hline
\end{tabular}

Fuente A.C.E. p. LXXXV

De esta lista destaca Van Camp Sea Food Co y Wilbur-Ellis que dominaba el mercado mundial. En el caso de las empresas peruanas destaca la empresa Carrillo que tenía agencias en Carquín, Huarmey, Samanco y Chimbote.

La materia prima era nacional pero se importaba la hojalata de EE.UU., para lo cual fue de gran utilidad la embajada peruana en Washington que era requerida por las empresas. ${ }^{2}$ En 1945 funcionaban 23 fábricas, 6 con

2 A M RR EE. 5-3-A/ 290 (21 de junio 1949). 
gran tamaño, y entre 1945 y 1948 se abren empresas en Ilo (Wilbur-Ellis) y en Chancay (Gildemeister). En medio de este crecimiento, la industria sufrió su primera crisis en 1948 al aparecer en el mercado de EE. UU. la producción japonesa, al afectar EE. UU. las importaciones de pescado y al rebajar el gobierno norteamericano la clasificación del producto peruano de «atún» a «bonito» (cf. Thorp y Bertram 1988: 371).

En 1949 el precio de los productos de pescado era el siguiente: $1 \mathrm{Tm}$ de conserva de pescado (6945 soles), 1 Tm pescado congelado (1119 soles) y de $1 \mathrm{Tm}$ de harina de pescado (814,5 soles) siendo el cambio de 1 dólar por 6,5 soles. $^{3}$

En 1950, el número de empresas pesqueras llegó a 49, de las cuales 15 eran consideradas grandes (cf.Thorp y Bertram 1988: 372). En esta década la producción pesquera peruana tuvo dificultades en el mercado de EE.UU. reorientándose las exportaciones en los años 1952-1954 hacia el mercado de Europa Occidental. Así, en 1956, mientras EE. UU. seguía comprando más de 7000 Tm de pescado congelado, el Reino Unido compró más de 7400 Tm de pescado en conservas Ante las dificultades que se presentaron en el mercado de EE.UU. se produjo una reorientación hacia Europa destacando Reino Unido, Holanda y Alemania en la década de $1950 .{ }^{4}$

En la década de 1950, la industria conservera peruana tuvo un gran desarrollo: llegó a producir 21000 Tm de conservas entre 1955-1959 (cf. Klaren 2004: 369), debido al crecimiento de la demanda de EE. UU., país embarcado en la Guerra Fría. No obstante, en 1958 la política arancelaria de EE. UU. cerró este mercado a la producción nacional, el sector de 69 fábricas pasó a sólo 10 a inicios de la década de 1960. El auge desde mediados de la década de 1950 se debió al crecimiento de la demanda mundial por alimentos para animales y la producción aumentó decididamente gracias a la adquisición de redes de nylon (cf. Thorp y Bertram 1988: 372-373).

3 Anuario del Comercio Exterior 1949: IX y XXXIX.

4 Anuario del Comercio Exterior 1956 : 112-114. 
En 1950 la primera fábrica dedicada a la reducción de anchoveta fue construida en Chimbote usando equipo de California «Pesquera Chimu» que fue una operación conjunta de la empresa Wilbur - Ellis y de Manuel Elguera empresario pesquero (cf. Roemer 1970: 83).

En la década de 1950 empresas peruanas, como la Compañía Peruana de Productos del Mar, solicitaban al Ministerio de Hacienda que por tener en Paita una planta congeladora y almacenes para pescado pueda recibir la captura de compañías extranjeras sin pagar derechos. ${ }^{5}$

Según el Boletín Estadístico Industrial del Ministerio de Fomento y Obras Públicas, las empresas pesqueras eran las siguientes:

\section{Empresas pesqueras 1954-1955}

\begin{tabular}{|c|c|c|c|}
\hline Año & Empresas & Trabajadores & $\begin{array}{c}\text { Mujeres } \\
\text { obreras }\end{array}$ \\
\hline 1954 & $\begin{array}{c}19 \text { (Lima - Callao) y } 9 \\
\text { (Provincias) }\end{array}$ & 3734 & 1664 \\
\hline 1955 & $\begin{array}{c}23 \text { (Lima Callao) y 12 } \\
\text { (Provincias) }\end{array}$ & 4259 & 1571 \\
\hline
\end{tabular}

Fuente B. E. I. 1954 y 1955

Estas cifras demuestran una disminución con el número de empresas de 1950, aunque el número de trabajadores aumentó destacándose el elevado porcentaje de la mujer trabajadora en este sector. ${ }^{6}$

La industria de la harina de pescado nació en el Perú en 1955 (cf. Zapata 2002: 356). El monto de la exportación de harina de pescado salto de $6600 \mathrm{Tm}$ a $18700 \mathrm{Tm}$ superando por primera vez a las exportaciones en conservadas y pescado congelado.

5 Memoria del Ministerio de Hacienda (1953: 232).

6 Ministerio de Fomento. Boletín de Estadística Industrial (1954: 52, 60, 71 y 76); (1955: 89-90). 
Según el Boletín Estadistica Industrial en 1956 existían las siguientes empresas

\begin{tabular}{|c|c|c|c|}
\hline Empresa & Lugar & $\frac{\text { Año }}{1090}$ & $\frac{\text { Trabajadores }}{221}$ \\
\hline Industria Pesquera & Callao & 1940 & \\
\hline $\begin{array}{l}\text { Compañía Conservas de } \\
\text { Pescados S. A. }\end{array}$ & Lima & 1942 & 44 \\
\hline $\begin{array}{l}\text { Sociedad Unión Pesquera y Envases } \\
\text { S.A. }\end{array}$ & Supe & 1944 & 198 \\
\hline Conservas y Salazones & Huacho & 1945 & 200 \\
\hline $\begin{array}{l}\text { Sociedad Industrial Conservas } \\
\text { Alimenticias }\end{array}$ & Chancay & 1945 & 33 \\
\hline Sindicato Pesquero Perú & Callao & 1945 & 60 \\
\hline Francisco C. Marfe & Callao & 1946 & 79 \\
\hline Industria Marina Alimentaria & Callao & 1947 & 36 \\
\hline Fabrica Conservas S. Casaretto & Rimac & 1946 & 38 \\
\hline Cia Marítima Pesquera S.A. & Chancay & 1948 & 436 \\
\hline Conservera Peruana & Callao & 1948 & 5 \\
\hline Francisca conservera América & Rimac & 1949 & 63 \\
\hline Mario Battilana & Chancay & 1951 & 24 \\
\hline Empresa Pesquera Peruana S.A. & Supe & 1953 & 222 \\
\hline Envasadora Rimac & S. M. Porres & 1954 & 60 \\
\hline Conservera Alimenticias S.A. & Huacho & 1954 & 35 \\
\hline Fabrica Conservera Gold Fish & Lima & 1956 & 35 \\
\hline Envases San Marcos & Lima & 1955 & 89 \\
\hline \multicolumn{4}{|l|}{ Ancash } \\
\hline Compañía Industrial Alimentos & Huarmey & 1945 & 4 \\
\hline Compañía Puerto Cosichco & Santa & 1945 & 491 \\
\hline Empresa Pesquera Chimu & Chimbote & 1950 & 82 \\
\hline Conservera Miramar & Chimbote & 1952 & 193 \\
\hline Inca Fishing & Chimbote & 1953 & 12 \\
\hline Andes Fishing Company & Chimbote & 1954 & \\
\hline Compañía Puerto La Florida & Chimbote & 1955 & 147 \\
\hline Envasadora Santa Rosa & Chimbote & 1955 & 98 \\
\hline Envasadora Chimbote & Chimbote & 1956 & 96 \\
\hline Bibaco S.A. & Chimbote & 1955 & 17 \\
\hline Compañía Industrial Peruana & Huarmey & 1955 & 40 \\
\hline Industria Pesquera Rosario & Chimbote & 1955 & 9 \\
\hline Industria Marítima & Casma & 1955 & 3 \\
\hline Compañía Marítima Pesquera & Chimbote & 1956 & \\
\hline Conservera Harinas Peruanas & Chimbote & 1956 & 2 \\
\hline \multicolumn{4}{|l|}{ Arequipa } \\
\hline Empresa Pesquera Mollendo & Islay & 1948 & 178 \\
\hline \multicolumn{4}{|l|}{ Moquegua } \\
\hline Compañía Pesca San Pedro & Ilo & 1942 & 180 \\
\hline & Ilo & 1945 & 731 \\
\hline
\end{tabular}




\begin{tabular}{|l|c|c|c|}
\hline Piura & & & \\
\hline Compañía Negocios Vitrano & Mancora & 1949 & 90 \\
\hline Frigorífico Paita & Paita & 1954 & 129 \\
\hline Frigorífico Pesquero Nor Peruano & Paita & 1955 & 61 \\
\hline Industria Marítima San Antonio & Paita & 1956 & 9 \\
\hline
\end{tabular}

Fuente B. E. I. 1956: 7-8

De estas empresas la Compañía Puerto Cosichco era subsidiaria de la Star Kist de Los Angeles (EE. UU.). De las empresas peruanas destacaba Compañía Puerto La Florida, fundada en 1955, de propiedad de Banchero Rossi. En Perú existían 50 empresas con 4447 trabajadores. En Lima y Callao se localizan 18 empresas, y en provincias 22, en Lima-Callao destaca que de los 1876 trabajadores más del $25 \%$ se ubicaban en Chancay, Supe y Huacho, y en Provincias de los 2571 trabajadores la ciudad de Chimbote contaba con el 23,9\% convirtiéndose en la capital de la industria pesquera peruana.

En 1955, el Perú producía poco menos de 16000 toneladas de harina de pescado al año, con un costo de producción de 55 dólares americanos por tonelada. En 1956, la producción se duplicó (32 000 toneladas), tendencia que continuó en la siguiente década.

El Perú, a diferencia de EE.UU. y del Japón, no desarrolló una industria para el consumo humano directo, sino se orientó al consumo animal aprovechando las características de la fauna pelágica donde predomina la anchoveta.

\section{AgroeXPortación y PESQUERÍA}

El guano que marcó la historia del siglo XIX no se consumió con la Guerra contra Chile (1879-1883), su explotación pasará a manos del estado peruano en el siglo XX que lo explota a favor del sector agroexportador, que tiene un gran desarrollo desde la Reconstrucción Nacional. En el siglo XX, en 1909, durante la República Aristocrática se creó la Compañía Administradora del Guano debido al agotamiento del guano fósil de las islas. La C. A. G. tenía el cuidado de las aves guaneras y la recolección del guano para ser empleado 
por la agricultura. Las aves guaneras se alimentaban de anchoveta y sardina. La C. A. G. contrató especialistas de EE. UU. durante el Oncenio para un mejor cuidado de las aves guaneras. Como resultado la población creció a 28 millones y se recolectó 300000 Tm de guano. Los agroexportadores obtenían así un abono, el guano, que permitiría el incremento de su producción. Esta situación, en la que se protegía y estudiaba a las aves guaneras, se deteriora desde la década de 1950 cuando por impacto de la industrialización, la gran demanda de fertilizante hace que el abono industrial desplace al guano y el alimento de las aves guaneras, anchoveta y sardina, sea más valioso que el guano.

En la década de 1950, la Compañía Administradora del Guano elabora una serie de informes que denuncian la actividad pesquera que afecta a las aves guaneras pero sin éxito.

Las fábricas conserveras nunca se consolidaron como una gran industria, pero permitieron crear los lazos que vincularon al Perú con EE. UU. para acceder así a una maquinaria como la fábrica de harina de gran utilidad en el Perú. Es paradójico cómo originalmente el gobierno pensó en la harina de pescado como fertilizante encomendando su producción a la Compañía Administradora del Guano que defendía el cuidado de las aves guaneras alimentadas por anchoveta y sardina, que pasarían a ser alimento de aves y mamíferos del mundo occidental (Abramovich: 1973).

\section{Conclusiones}

La industria pesquera de EE. UU. explotó intensamente sus recursos marinos durante 20 años después de la Primera Guerra Mundial y los agotó hacia fines de la década de 1940 .

La industria pesquera de EE.UU. extendió sus operaciones hacia el sur del Océano Pacífico, desde México hasta Chile, desde la década de 1930 y especialmente después de la Segunda Guerra Mundial.

Los altos costos de producción en EE.UU. llevaron a vender fábricas de harina de pescado a otros países como Perú. 
RECURSOS INFORMATIVOS

Fuentes Primarias

AGN Ministerio de Agricultura Resoluciones Directoriales 1944

Archivo del Ministerio de Relaciones Exteriores: Sección Perú- EE. UU. (53-A) 1947- 1956.

\section{IMPRESOS}

Dirección General de Aduanas (1945), Anuario de Comercio Exterior 1944. Callao: Dirección General de Aduanas.

Ministerio de Hacienda y Comercio (1947), Anuario de Comercio Exterior II Parte 1945- 1946, Callao: MHC.

Ministerio de Hacienda y Comercio (1950), Memoria, Lima: MHC.

Ministerio de Hacienda (1950), Anuario de Comercio Exterior 1949, Lima: MHC.

Ministerio de Hacienda y Comercio (1950), Anuario de Comercio Exterior 1949 Callao: MHC.

Ministerio de Hacienda y Comercio (1951), Anuario de Comercio Exterior 1950 Callao: MHC.

Compañía Administradora del Guano (1952), Memoria 1951, Lima: CAG. Compañía Administradora del Guano (1953), Memoria 1952, Lima: CAG. Ministerio de Hacienda y Comercio (1953), Memoria, Lima: MHC.

Ministerio de Hacienda (1955), Boletín Estadística Industrial 1955, Lima: MHC. 


\section{ORÍGENES DE LA INDUSTRIA PESQUERA PERUANA}

Boletín Oficial Dirección de Industrias y electricidad (1956), Padrón de Industrias Manufactureras, Lima.

Ministerio de Hacienda y Comercio (1957), Estadística del Comercio Exterior 1956, Lima: Ministerio de Hacienda y Comercio.

Ministerio de Hacienda. Boletín (1958), Estadístico Industrial 1954, Lima, MHC. 


\section{BIBLIOGRAFÍA}

ABramovich, J.

1973 La industria pesquera en el Perú, Apogeo y Crisis. Lima: Imprenta Popular.

Adrianzén Merino, Luis Alberto

1980 Relaciones internacionales Perú-EE.UU. Lima: PUCP.

Alonso Aguilar, Chris Reid y Andy Thorpe

2000 «The Political Economy of Marine Fisherie Development in Peru Chile y Mexico». Journal of Latino American Studies 32(2), pp. 503-527.

Carey, James C.

1964 Peru and the United States, 1900-1962. University of Notre Dame Press.

Clayton, Lawrence A.

2002 Estados Unidos y el Perú: 1800-1995. Lima: Instituto Peruano de Economía Social de Mercado y CEPEI.

Contreras, Carlos y Marcos Cueto

1999 Historia del Perú Contemporáneo. Lima: Red de Desarrollo de las Ciencias Sociales.

ECKes, A. E.

1992 «Trading American interests». Foreign Affairs 71(4), pp.135-54.

Ferrero Costa, Eduardo (ed.)

1987 Relaciones del Perú con los Estados Unidos. Lima CEPEI. 
FINLEY, Mary Carmel

2007 The Tragedy of Enclosure: Fish, Fisheries Science, and U.S. Foreign Policy, 1920-1960. A Dissertation submitted in partial satisfaction of the requirements for the degree Doctor of Philosophy University of California, San Diego.

2009 «The Social Construction of Fisherie, 1947». Ecology and Society 14:1. <www.ecologyandsociety.org/vol14/iss1/art6/>.

KLAREN, Peter

2004 Nación y sociedad en el Perú. Lima: IEP.

LORING, David

1971 «The United States Peruvian Dispute». Stanford Law Review 23(3), 391- 453.

MaceVoy, Arthur

1983 «Law Public Policy and Industrialization in the California Fisheries 1900-1925». Business History Review 57, pp. 494-521.

1990 The fisherman Problem: Ecology and Law in California Fisheries 18501980. Cambridge: University Press: Cambridge.

Oreskes, Naomi y Carmen FinLeY

2007 «A Historical Analysis of the Collapse of Pacific Groundfish: U.S. Fisheries Science, Development, and Management, 19451995" Research Completion Reports (University of California, San Diego) <http://repositories.cdlib.org/cgi/viewcontent.cgi?article $=1158 \&$ context $=\operatorname{csg} \mathrm{c}>$.

Petit, Thomas A.

1966 «Import of import and tariffs in the American Tuna Industry». American Journal of Economic and Society 19(3): 275-288. 
PIKE, Frederik B.

1977 The United States and the Andean Republics: Peru, Bolivia, and Ecuador. Cambridge Massachussets.

Roemer, Michael

1970 Fishing for growth export led Development in Perú 1950-1967. Harvard University Press.

Shimizu, Sayuri

2001 Creating People of plenty The United States and Japan's Economics Alternatives, 1950-1960. Kent and London: Kent University Press.

ThOrp, Rosemary y G. BERTRAM

1988 Perú: 1890-1977. Crecimiento y políticas en una economía abierta. Lima: Mosca Azul.

Zapata, Antonio

2002 «Los actores y la problemática de la pesca industrial peruana 19551997. En Glave, L. M. y C. Contreras. Estado y mercado en la Historia del Perú. Lima: IEP. 


\section{ANEXOS}

\section{Exportación en Tm}

\begin{tabular}{|l|l|l|l|l|l|l|l|}
\hline & 1943 & 1944 & 1945 & 1946 & 1949 & 1950 & 1956 \\
\hline Pescado Conservas & 962 & 914,9 & 1753,6 & 2652,5 & 6199 & 8721 & 17757 \\
\hline Pescado congelado & & 1772,8 & 4085 & 7040,2 & 6212 & 9106 & 13315 \\
\hline Harina de pescado & 534 & 738 & 694,8 & 553,7 & 2708 & 3742 & 27792 \\
\hline Tripa & 270 & 182 & 170 & & & & \\
\hline Aceite & & & 117,5 & & & & 5750 \\
\hline Total & 1766 & 3607,7 & 6820,9 & 10246,4 & 11508,1 & 21659 & 64614 \\
\hline
\end{tabular}

Fuente: Estadística de Comercio Exterior (1944, 1946, 1950 y 1956)

Valor en soles (en miles de soles)

\begin{tabular}{|l|r|r|r|r|r|r|r|}
\hline & 1943 & 1944 & 1945 & \multicolumn{1}{c|}{1946} & 1949 & 1950 & 1956 \\
\hline $\begin{array}{l}\text { Pescado } \\
\text { conserva }\end{array}$ & $2380,3^{*}$ & 2623,1 & 4988,1 & 8081,6 & 43049,9 & 58501 & 92357,0 \\
\hline $\begin{array}{l}\text { Pescado } \\
\text { congelado }\end{array}$ & 22,3 & 236,8 & 217,9 & 267,5 & 2206,0 & 4250 & 67211,0 \\
\hline $\begin{array}{l}\text { Harina de } \\
\text { Pescado }\end{array}$ & 87,4 & 59,4 & 1316,6 & 4000,7 & 6959,7 & 22762 & 35,1 \\
\hline Tripa & 2690, & 3537,7 & 6522,6 & 12086,3 & 52215,6 & 1040252 & 159620,4 \\
\hline Aceite & & & & & & 17,3 \\
\hline Total & & & & & & & \\
\hline
\end{tabular}

Fuente: Estadística de Comercio Exterior (1944, 1946, 1950 y 1956)

* Considera Pescado Congelado y en Conservas 
Flota Pesquera del Perú 1950 - 1956

\begin{tabular}{|l|l|l|l|l|}
\hline Años & N. ${ }^{\circ}$ de barcos & Bodega total & $\begin{array}{c}\text { N. }{ }^{\circ} \text { de } \\
\text { plantas }\end{array}$ & \multicolumn{1}{|c|}{ Capacidad Tm/hora } \\
\hline 1950 & 25 & 806 & 1 & 5 \\
\hline 1951 & 50 & 1863 & 8 & 75 \\
\hline 1952 & 75 & 3169 & 14 & 146 \\
\hline 1953 & 100 & 4728 & 21 & 216 \\
\hline 1954 & 126 & 6586 & 27 & 287 \\
\hline 1955 & 175 & 10,020 & 34 & 397 \\
\hline
\end{tabular}

Fuente: Zapata: 2002: 404 